\title{
Laparoscopic abdominoperineal resection with intraoperative radiotherapy for locally advanced low rectal cancer
}

\author{
Matej Skrovina a, , Renata Soumarovab ${ }^{\mathrm{b}}$, Miloslav Duda ${ }^{\mathrm{a}, \mathrm{d}}$, Roman Bezdek ${ }^{\mathrm{e}}$, Jiri Bartos ${ }^{\mathrm{a}}$, Adam Wendrinski ${ }^{\mathrm{b}}$, Petr Andel ${ }^{\mathrm{a}}$, \\ Javed Parvez ${ }^{\mathrm{a}}$, Martin Straka ${ }^{\mathrm{a}}$, Lukas Adamcik ${ }^{\mathrm{a}}$
}

\begin{abstract}
Aims. Intraoperative radiotherapy (IORT) for locally advanced rectal cancer as an integral part of multimodal treatment, may lead to reduced local recurrence but it is not routinely used. The aim of this paper is to describe our experience with IORT in the treatment of patients with locally advanced adenocarcinoma of the lower third of the rectum.

Material and Methods. Laparoscopic abdominoperineal amputation of the rectum with intraoperative radiotherapy was performed on 17 patients, 13 men and 4 women, median age 64 years (49-75 years) between 2010-2011. All patients underwent complete therapy according to the treatment protocol.

Results. In one patient, the laparoscopic procedure had to be converted to an open resection. The duration of the surgical procedure with IORT was 185 to $345 \mathrm{~min}$ (median $285 \mathrm{~min}$ ). In 14 cases, the intraoperative dose was 10 Gy and in two patients a dose of $12 \mathrm{~Gy}$ was used. There were no severe intraoperative complications. Blood loss ranged from 30 to $500 \mathrm{~mL}$ (median $100 \mathrm{~mL}$ ). There were postoperative complications in 4 patients (23.5\%); 2 necessitated surgical reintervention (11.8\%). The duration of postoperative hospitalization was 6 to 35 days (median 7 days). In the follow-up of 2 to 16 months (median 12 months), no local recurrence or disease generalization have been found to date.

Conclusions. The results show the technical feasibility of laparoscopically assisted abdominoperineal amputation of the rectum in combination with IORT in the treatment of locally advanced rectal carcinoma with an acceptable risk of postperative complications.
\end{abstract}

Key words: rectal cancer, surgery, intraoperative radiotherapy, laparoscopy

Received: February 29, 2012; Accepted: July 25, 2012; Available online: November 2, 2012

http://dx.doi.org/10.5507/bp.2012.082

${ }^{a}$ Department of Surgery, Hospital and Oncological Centre, Novy Jicin, Czech Republic

${ }^{b}$ Department of Oncology and Radiotherapy, Hospital and Oncological Centre, Novy Jicin

'Department of Surgery I, University Hospital Olomouc and Faculty of Medicine and Dentistry, Palacky University Olomouc

${ }^{d}$ Department of Surgery II, University Hospital Olomouc and Faculty of Medicine and Dentistry, Palacky University Olomouc

${ }^{e}$ Department of Anesthesiology, Hospital and Oncological Centre, Novy Jicin

Corresponding author: Matej Skrovina, e-mail: matej.skrovina@nemnj.cz

\section{INTRODUCTION}

Local recurrence in patients with rectal cancer remains a serious problem. The incidence after radical operation ranges from 2.6 to $32.0 \%$ and it has a very poor prognosis ${ }^{1,2}$. In patients with total mesorectal excision and an achieved negative radial resection margin (circumferential resection margin) local recurrence is less than $10 \%$. However, up to $28 \%$ of patients with total mesorectal excision have findings of a positive radial resection margin (less than $1 \mathrm{~mm}$ ). In these patients the risk of local recurrence dramatically rises (up to $38 \%$ ) (ref., ${ }^{3,4}$ ). Due to its high risk, treatment of locally advanced rectal cancer requires an individualized approach, including optimalization of surgical technique and deciding on possible multimodal treatment. Part of this approach includes preoperative or postoperative external radiotherapy. Due to better oncological and functional results, preoperative radiotherapy is generally preferred, often in combination with concomitantly applied chemotherapy ${ }^{5,6}$. Patients with a positive or very narrow radial resection margin require radiotherapy in a dose exceeding $60 \mathrm{~Gy}$ to achieve better local control. Administration of such a high dose of external radiotherapy exceeds the tolerated dose and carries a high risk of causing damage to surrounding organs, primarily the small intestine and the urinary bladder. A solution seems to be the combination of preoperatively administered radiotherapy in a dose of 45.0 -50.4 Gy and radical resection with application of intraoperative radiotherapy to a precisely defined area while protecting the surrounding structures ${ }^{7,8}$.

\section{MATERIAL AND METHODS}

\section{Patient sample}

Patients with precisely defined indication criteria were indicated for IORT. All had histologically verified primary locally advanced adenocarcinoma of the lower rectumstage T3-4NxM0 (International Union Against Cancer, 7th edition), had no known oncological disease in their medical history, had never undergone radiotherapy to the pelvic region and were never administered chemotherapy. As part of disease staging, all patients underwent pancolonoscopy to rule out possible synchronous colorectal cancer, chest X-ray or CT, abdominal CT, and MRI of 
the pelvic region. Patients with a very severe comorbidity and those younger than 18 years were not indicated for this treatment. Prior to the beginning of therapy, all patients were reviewed by a multidisciplinary oncological committee and gave written consent to the planned treatment procedure.

\section{Treatment protocol}

All patients were indicated for neoadjuvant concomittant chemoradiotherapy. External radiotherapy was applied to the pelvic area in 25 fractions ( 5 fractions/week) totaling a dose of $45 \mathrm{~Gy}$ (1.8 Gy/fraction). Together with the radiotherapy, patients were administered capecitabin in a peroral dose of $825 \mathrm{mg} / \mathrm{m}^{2}$ every $12 \mathrm{~h}$. The surgical procedure was planned 6-8 weeks after the completion of chemoradiotherapy. The day before the surgery, all patients underwent an MRI scan of the pelvic region. Patients selected for IORT were those patients with findings of persisting T3-4 tumor (International Union Against Cancer, 7. edition), in which the MRI showed suspicion of possible infiltration to the mesorectal fascia or surrounding organs - T3cd, T4ab (ref., ${ }^{9}{ }^{10}$ ). Patients with sphincter-preserving or multivisceral resection were not included, only patients with laparoscopically assisted abdominoperineal amputation of the rectum were selected. One port for the laparoscopic camera and three working ports were used during the resection in all patients. At the beginning of the operation, an intraoperative ultrasound of the liver was performed to rule out possible preoperatively occult synchronous metastases. The abdominoperineal amputation of the rectum with reconstruction of the perineum followed. After completing the resection and selecting a suitable tubus, the patient was transported to the

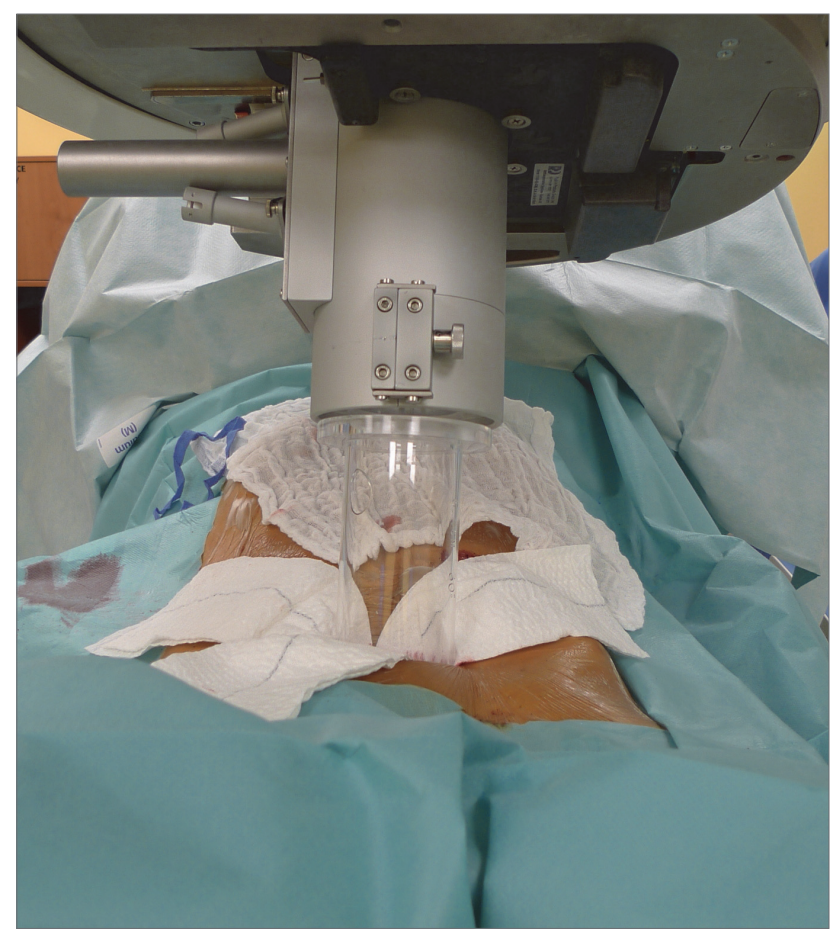

Fig. 1. IORT - connecting the tubus to the head of the linear accelerator. radiation treatment room while continually monitoring vital functions. Via suprapubically localized minilaparotomy (Phannestiel incision), a tubus was introduced into the presacral area, which after isolation of surrounding structures was attached to the radiating head of the linear accelerator (Fig. 1). The dose was determined based on the completeness of the surgical resection taking into account the possibility of a positive radial resection margin. Standardly, in cases of R0 resection, a dose of $10 \mathrm{~Gy}$ is administered; if an R1 resection is presumed 12 Gy are administered, and if macroscopic tumor residuum remains (R2) then $15-20$ Gy are administered ${ }^{11-13}$. Following radiation, the patient was transported back to the operating room where the surgical procedure was completed (Fig. 2). Adjuvantly, patients were again administered 4-6 cycles of capecitabin in a dose of $825 \mathrm{mg} / \mathrm{m}^{2}$ perorally every $12 \mathrm{~h}$. All patients underwent follow-up visits on the $7^{\text {th }}$ and $30^{\text {th }}$ day after being released from hospital and then every 3 months where follow up examinations, as part of oncosurgical dispensarisation, took place.

Between August 31st, 2010 and November14th, 2011, a total of 212 patients with colorectal cancer were operated on at the Department of Surgery at the Hospital Nový Jičin. Eighty of these patients had tumors localized less than $12 \mathrm{~cm}$ from the anal verge. Of these patients, 17 with locally advanced primary adenocarcinoma of the lower rectum were selected for abdominoperineal amputation of the rectum with intraoperative radiotherapy. The set included 13 men and 4 women with a median age of 64 years (49-75 years). The median body mass index was $28.1 \mathrm{~kg} / \mathrm{m}^{2}\left(18.1-34.2 \mathrm{~kg} / \mathrm{m}^{2}\right)$. All patients underwent complete therapy according to the treatment protocol. The distance of the lower border of the tumor from the anal

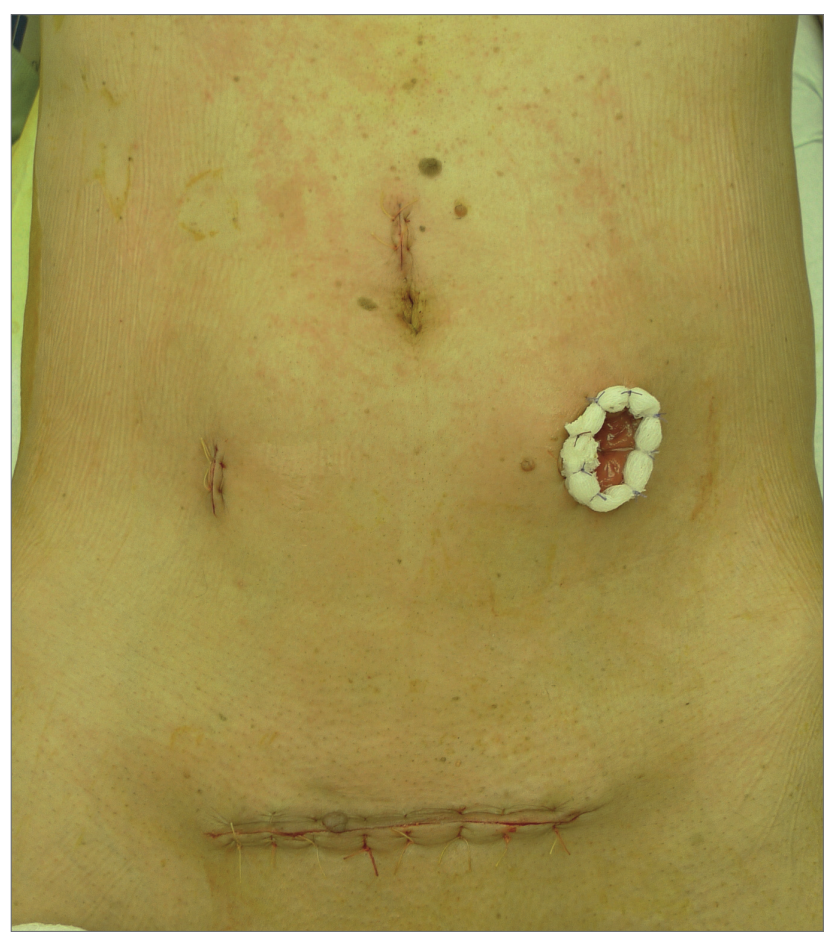

Fig. 2. IORT - placement of incisions for laparoscopic ports, terminal colostomy and mini-laparotomy fro tubus insertion. 
verge was $25 \mathrm{~mm}(15-30 \mathrm{~mm})$ and the surgical procedure was performed 41 to 138 days (median 49 days) after the completion of neoadjuvant chemoradiotherapy. The operation performed 138 days after completing preoperative oncological treatment was due to postponement for personal reasons by the patient.

\section{RESULTS}

Sixteen of the 17 patients included in the study underwent laparoscopically assisted abdominoperineal amputation of the rectum. In one patient with a BMI of $34.2 \mathrm{~kg} / \mathrm{m}^{2}$, the surgical procedure was converted to an open procedure during the phase of mesorectal excision. The duration of surgical procedure together with IORT was 185 to $345 \mathrm{~min}$ (median $285 \mathrm{~min}$ ). The duration of IORT alone, including transportation of the patient to the radiation treatment room and back was 22 to 35 min (median $25 \mathrm{~min}$ ). In all operated patients, the tubus used for IORT had an angle of $30^{\circ}$, in 16 patients the diameter was $64 \mathrm{~mm}$ and in one patient with a narrow pelvis a tubus with a diameter of $32 \mathrm{~mm}$ was used. In 14 patients the intraoperative dose administered was 10 Gy. In two patients a dose of 12 Gy was administered, in one of these patients definitive histological findings confirmed microscopic tumorous infiltration of the circumferential resection margin $(<1 \mathrm{~mm})$. No serious intraoperative complications were observed in any of the patients. Blood loss ranged from 30 to $500 \mathrm{~mL}$ (median $100 \mathrm{~mL}$ ). Postoperative complications were observe in four patients $(23.5 \%)$, of those only two required surgical reintervention (11.8\%). A 59-year-old patient was re-operated on the $3^{\text {rd }}$ postoperative day for ileus of the small intestine, caused by incarceration of a loop of the terminal ileum in a small opening in the pelvic peritoneal suture line. Re-operation, however, did not require resection of the small intestine, and the defect in the suture of the peritoneum was treated by resuture. One patient presented with an abscess of the perineum and presacral space, which was treated by application of the negative pressure wound therapy system. This patient in the later postoperative course had ongoing urinary retention which required long-term derivation by urinary catheter. In one patient a small hematoma was evacuated from the incision on the perineum and in one patient a subcutaneous hematoma around the colostomy was treated conservatively. The duration of postoperative hospitalization was 6 to 35 days (median 7 days). All patients are currently followed up at our department and in a follow-up interval of 2 to 16 months (median 12 months). No local recurrence or disease generalization have been observed to date.

\section{DISCUSSION}

Intraoperative radiotherapy, as a part of multimodal therapy, improves local control in patients with locally advanced or recurrent cancer of the rectum ${ }^{14,15}$. The risk of local recurrence in the area where radiotherapy was applied is lower in IORT when compared with external radiotherapy. Local control is $8 \%$ higher when using IORT (ref. ${ }^{13,16-20}$ ). Roeder and Krempien actually report up to $18 \%$ lower incidence of local recurrence in the area of applied IORT (ref. ${ }^{13,20}$ ). Sadahiro et al. in their study aimed at patients with advanced rectal cancer T3-4NxM0, compared achieved results between a group of 68 patients with resection alone and a group of 99 patients where radiochemotherapy was applied and subsequently resection with IORT was performed. Both groups were comparable in age, gender, tumor localization, tumor size and stage. $44 \%$ of patients with primary resection and $53 \%$ of patients with an initially multimodal approach underwent postoperative chemotherapy. Five-year overall survival was $79 \%$ in patients with combined therapy versus $58 \%$ in patients who underwent only surgical resection ${ }^{12}$. In our small sample of patients, no cases of local recurrence after IORT have been reported to date. However, the duration of follow-up is too short (median 12 months) for conclusions to be drawn.

Generally IORT is associated with a higher risk of perioperative complications and a serious postoperative complication rate of $30-40 \%$. Most often these include gastrointestinal, urological and dermatological complications. We frequently encounter infectious complications associated with the surgical procedure in patients after IORT (ref. ${ }^{21}$ ). Mathis et al. reported a significant association between radiation dose and frequency of pelvic complications. A lower incidence of these complications was noted with doses of less than 12.5 Gy (ref. ${ }^{16}$ ). DiazGonzalez et al. presented up to $37 \%$ morbidity associated with IORT as part of multimodal therapy. The most frequent complications were surgical site infection, pain and presacral abscess ${ }^{17}$. Sadahiro et al. reported 23\% surgical site infections in patients with IORT in comparison to $11.7 \%$ complications in patients who underwent only the surgical resection ${ }^{12}$. Mannaerts et al. noted 29\% surgical site infections associated with IORT administration as part of a multimodal treatment of rectal cancer ${ }^{22}$. We found an infectious complication in the form of an abscess in the presacral space and in the perineal incision in one patient $(5.9 \%)$. A possible decrease in the incidence of surgical site infections in our pilot study may be associated with the use of a minimally-invasive approach, and laparoscopic assistance during abdominoperineal amputation of the rectum.

It is often reported in the literature that IORT administration is associated with a higher risk of postoperative urological and gastrointestinal complications. Bladder malfunction is reported in 2 to $16 \%$ of cases and postoperative ureteral obstruction in up to $12 \%$ (ref. ${ }^{12,16,20,22,23}$ ). In our set of patients, postoperative urinary retention was encountered in one patient $(5.9 \%)$, which required drainage by long-term indwelling catheter. Gastrointestinal complications are reported in up to $14 \%$ of cases ${ }^{16}$. Another complication which may be associated with IORT application is peripheral neuropathy, which is seen in $8-19 \%$ of cases $^{16,22}$. 
Combining laparoscopic resection of the rectum with IORT is very rare to date. Civello et al. presented a sample of six patients with locally advanced rectal cancer, who underwent neoadjuvant radiochemotherapy followed by laparoscopic resection of the rectum with IORT. The duration of the surgical procedure ranged from 280 to 360 min. There were no severe complications associated with the operation and all patients were released from hospital by the $8^{\text {th }}$ postoperative day ${ }^{24}$.

\section{CONCLUSION}

Intraoperative radiotherapy of primary rectal cancer as part of a multimodal therapy leads to a decrease in the incidence of local recurrence, however, with an acceptably slightly higher risk of postoperative infectious complications. Long-term follow-up of these selected patients as well as comparison of the results are therefore necessary to establish definite guidelines.

\section{ACKNOWLEDGEMENTS}

Authorship contributions: MSk, PA, LA: literature search; MSk, RB, AW: data collection; MSk, JB, JP: data analysis; MSk, MSt: data interpretation; MSk: manuscript writing; MSk, RS, MD: final approval.

Conflict of interest statement: The authors stated that there are no conflicts of interest regarding the publication of this article.

\section{REFERENCES}

1. Heriot A, Kumar D. Rectal cancer recurrence: factors and mechanisms. Colorectal Dis 2000;2:126-37.

2. McCall JL, Cox MR, Wattchow DA. Analysis of local recurrence rates after surgery alone for rectal cancer. Int J Colorectal Dis 1995;10:12632.

3. Nagtegaal ID, Marijnen CA, Kranenbarg EK, van de Velde CJ, van Krieken JH; Pathology Review Committee; Cooperative Clinical Investigators. Circumferential margin involvement is still an important predictor of local recurrence in rectal carcinoma: No tone milimeter but two millimeters is the limit. Am J Surg Pathol 2002;26:350-7.

4. Nagtegaal ID, Quirke P. What is the role for the circumferential margin in the modern treatment of rectal cancer? J Clin Oncol 2008;26:303-12.

5. Colorectal Cancer Collaborative Group. Adjuvant radiotherapy for rectal cancer: a systematic overview of 8,507 patients from 22 randomised trials. Lancet 2001;358:1291-304.

6. Sauer R, Becker H, Hohenberger W, Rodel C, Wittekind C, Fietkau R, Martus P, Tschmelitsch, Hager E, Hess CF, Karstens JH, Liersch T, Schmidberger H, Raab R; German Rectal Cancer Study Group. Preoperative versus postoperative chemoradiotherapy for rectal cancer. N Engl J Med 2004;351:1731-40.

7. Kang MK, Kim MS, Kim JH. Intraoperative radiotherapy for locally advanced rectal cancer. J Korean Soc Coloproctol 2010;26:274-8.

8. Soumarova R, Skrovina M, Machala S, Adamcik L, Bartos J. Intraoperative radiotherapy in multimodality treatment of locally advanced rectal carcinoma. Rozhl Chir 2010;89:685-8.
9. MERCURY Study Group. Extramural depth of tumor invasion at thinsection MR in patients with rectal cancer: results of the MERCURY study. Radiology 2007;243:132-9.

10. Glimelius B, Påhlman L, Cervantes A; ESMO Guidelines Working Group, Rectal cancer: ESMO Clinical Practice Guidelines for diagnosis, treatment and follow-up. Ann Oncol 2010;21 Suppl 5:v82-6.

11. Haddock MG, Nelson H, Donohue JH, Taylor WE, Devine RM, Wolff BG, O'Connell MJ, Gunderson LL. Intraoperative electron radiotherapy as a component of salvage therapy for patients with colorectal cancer and advanced nodal metastases. Int J Radiat Oncol Biol Phys 2003;56:966-73.

12. Sadahiro S, Suzuki T, Ishikawa K, Fukasawa M, Saguchi T, Yasuda S, Makuuchi H, Murayama C, Ohizumi Y. Preoperative radio/chemoradiotherapy in combination with intraoperative radiotherapy for T3-4Nx rectal cancer. Eur J Surg Oncol 2004;30:750-8.

13. Roeder F, Treiber M, Oertel S, Dinkel J, Timke C, Funk A, GarciaHuttenlocher $\mathrm{H}$, Bischof M, Weitz J, Harms W, Hensley FW, Buchler MW, Debus J, Krempien R. Patterns of failure and local control after intraoperative electron boost radiotherapy to the presacral space in combination with total mesorectal excision in patients with locally advanced rectal cancer. Int J Radiat Oncol Biol Phys 2007;67:1381-8.

14. Skandarajah AR, Lynch AC, Mackay JR, Ngan S, Heriot AG. The role of intraoperative radiotheraphy in solid tumors. Ann Surg Oncol 2009;16:735-44.

15. Cantero-Munoz P, Urien MA, Ruano-Ravina A. Efficacy and safety of intraoperative radiotherapy in colorectal cancer: $A$ systematic review. Cancer Letters 2011;306:121-33.

16. Mathis $\mathrm{KL}$, Nelson $\mathrm{H}$, Pemberton JH, Haddock MG, Gunderson LL. Unresectable colorectal cancer can be cured with multimodality therapy. Ann Surg 2008;248:592-8.

17. Diaz-Gonzales JA, Calvo FA, Cortes J, Garcia-Sabrido JL, Gomez-Espi $M$, Del Valle E, Munoz-Jimenez F, Alvarez E. Prognostic factors for disease-free survival in patients with T3-4 or N+ rectal cancer treated with preoperative chemoradiation therapy, surgery, and intraoperative irradiation. Int J Radiat Oncol Biol Phys 2006;64:1122-8.

18. Calvo FA, Gomez-Espi M, Diaz-Gonzales JA, Alvarado A Cantalapiedra R, Marcos P, Matute R, Martinez NE, Lozano MA, Herranz R. Intraoperative presacral electron boost following preoperative chemoradiation in T3-4Nx rectal cancer: initial local effects and clinical outcome analysis. Radiother Oncol 2002;62:201-6.

19. Kusters M, Holman FA, Martijn H, Nieuwenhuijzen GA, Creemers GJ, Daniels-Gooszen AW, van den Berg HA, van den Brule AJ, van de Velde CJ, Rutten HJ. Patterns of local recurrence in localy advanced rectal cancer after intra-operative radiotherapy containing multimodality treatment. Radiother Oncol 2009;92:221-5.

20. Krempien R, Roeder F, Oertel S, Roebel M, Weitz J, Hensley FW, Timke C, Funk A, Bischof M, Zabel-Du Bois A, Niethammer AG, Eble MJ, Buchler MW, Treiber M, Debus J. Long-term results of intraoperative presacral electron boost radiotherapy (IOERT) in combination with total mesorectal excision (TME) and chemoradiation in patients with locally advanced rectal cancer. Int J Radiat Oncol Biol Phys 2006;66:1143-51.

21. Valentini V, Aristei C, Glimelius B, Minsky BD, Beets-Tan R, Borras JM, Haustermans K, Maingon P, Overgaard J, Pahlman L, Quirke P, Schmoll HJ, Sebag-Montefiore D, Taylor I, Van Custem E, Van de Velde C, Cellini N, Latini P, on behalf of the Scientific Committee. Multidisciplinary rectal cancer management: 2nd European rectal cancer consensus conference (EURECA-CC2). Radiother Oncol 2009;92:148-163.

22. Mannaerts GH, Martijn H, Crommelin MA, Dries W, Repelaer van Driel OJ, Rutten HJ. Feasibility and first results of multimodality treatment, combining EBRT, extensive surgery, and IOERT in locally advanced primary rectal cancer. Int J Radiat Oncol Biol Phys 2000;47:425-33.

23. Kienle $P, A$ bend F, Dueck M, Abel U, Treiber M, Riedl . Influence of intraoperative and postoperative radiotherapy on functional outcome in patients undergoing standard and deep anterior resection for rectal cancer. Dis Colon Rectum 2006;49:557-67.

24. Civello IM, Cavicchioni C, Tacchino RM, Matera D, Valentini V, Manfrida S, Greco F. Laparoscopic resection with intraoperative radiotherapy: a new step in the multimodal treatment of advanced colorectal cancer. Surg Endosc 2007;21:330-2. 